\title{
Résonances et dissonances
}

Une pierre jetée dans l'eau fait des vagues. Simple vérité qui, au sens figuré, peut s'appliquer aux articles du BMS, comme c'est le cas maintenant déjà, dès les premières semaines de la nouvelle année. Trois articles sur la médecine complémentaire ont provoqué des réactions brièvement commentées ci-après. Il s'agit de la prise de position de la commission spécialisée de la Société suisse d'allergologie et d'immunologie (SSAI) sur la biorésonance [1], de l'article de Walter Buschauer sur la médecine expérimentale opposée à la médecine «hippocratique» [2], ainsi que des réflexions de Hans Geiser sur le programme d'évaluation des médecines complémentaires (PEK) [3].

On ne s'étonnera guère du fait que ces sujets aient d'emblée provoqué quelques craintes, exprimées lors de rencontres personnelles. «Est-ce le début d'une campagne contre la médecine complémentaire?» se demande un confrère médecin de premier recours au bénéfice d'une attestation de formation complémentaire en médecine chinoise (MTC), qui s'inquiète à propos de l'article sur la biorésonance (il n'avait vraisemblablement pas lu la colonne «Et encore...» du premier Bulletin des médecins suisses de cette année, ce dont on peut l'excuser, bien sûr). Une opinion contraire est exprimée par un confrère interniste enseignant dans un hôpital universitaire, qui s'insurge: «Comment pouvez-vous, en tant que bulletin des médecins, offrir une tribune à un homéopathe qui défend une doctrine allant à l'encontre de tout fondement scientifique?»

Ces réactions exemplaires suggèrent la réflexion suivante: si le Bulletin des médecins suisses est avant tout l'organe officiel de la FMH laquelle décerne par ailleurs les attestations de formation complémentaires en homéopathie - il a aussi la fonction d'un forum d'information pour tous les milieux intéressés à la santé publique et y participant. En tant qu'importante société de discipline, la SSAI peut évidemment prendre position dans le BMS sur tout sujet qui lui paraît important, donc également sur la biorésonance.

A propos de cette dernière méthode, qu'on me permette une remarque personnelle: lorsqu'une démarche médicale-qu'elle relève de la médecine universitaire ou complémentaire - se réclame de fondements scientifiques, il est impératif qu'elle se confronte aussi aux données les plus récentes de la science. La biorésonance exploite les possibilités de la technologie moderne ou à tout le moins, telle est son ambition. Les défenseurs à tout crin de ce qu'il est convenu d'appeler la médecine classique ne sont pas les seuls à postuler que ce faisant, elle interprète la physique de façon erronée et ne mesure pas ce qu'elle estime mesurer: des physiciens qui étudient les méthodes de médecine complémentaire dans une optique à la fois intéressée et bienveillante - tels Ulrich Warnk qui enseigne à l'Université de la Saare - parviennent en effet aux mêmes conclusions [4].

En revanche, recommander ou condamner en bloc l'ensemble de la médecine complémentaire me paraît aussi insensé que de déclarer tous les champignons comestibles ou vénéneux. C'est pourtant là une façon de juger relativement répandue, qu'illustre l'exemple de l'homéopathie, qui est sans aucun doute la discipline de médecine complémentaire suscitant les prises de position les plus contrastées. On le sait, il n'existe pas à ce jour de modèle scientifique établi permettant d'expliquer de façon satisfaisante le mode de fonctionnement des médicaments homéopathiques. En conclure, de façon purement théorique, que ceux-ci sont forcément inefficaces ne procède toutefois pas d'une attitude que l'on puisse qualifier de scientifique. Le défi consiste bien plutôt, aujourd'hui comme naguère, à trouver des méthodes d'évaluation de l'efficacité desdits médicaments qui soient scientifiquement fondées tout en tenant compte des particularités de l'homéopathie.

Il est intéressant de relever que Hahnemann, le fondateur de l'homéopathie, qualifiait les médecins «classiques» de son temps de charlatans et leur pensée de peu scientifique: aujourd'hui, c'est à ses disciples que l'on adresse ces mêmes reproches. Il préconisait un art de guérir rationnel fondé sur des observations exactes et en fit la démonstration en pratiquant sur lui-même un test avec de l'écorce de quinquina. L'absorption de cette substance ayant produit chez lui des symptômes semblables à ceux du paludisme, il en déduisit son fameux principe de similitude («similia similibus curantur»). Aujourd'hui encore, la médecine fondée sur les sciences naturelles continue à utiliser des préparations à base de quinine pour traiter le paludisme. N'avons-nous pas là un point de départ commun à partir duquel on pourrait répéter l'expérience, mais dans des conditions dûment contrôlées bien entendu?

Bruno Kesseli, rédacteur en chef Verlags-Gesellschaft; 1999. S. $50-2$ 